\title{
Determinanten der Länderwahl privater schweizerischer Entwicklungshilfe
}

\section{Christoph Stamm}

\section{(2) OpenEdition}

1 Journals

Electronic version

URL: http://journals.openedition.org/sjep/499

DOI: 10.4000/sjep.499

ISSN: 1663-9677

Publisher

Institut de hautes études internationales et du développement

Printed version

Date of publication: 1 novembre 2004

Number of pages: 87-104

ISBN: 2-88247-057-6

ISSN: $1660-5926$

\section{Electronic reference}

Christoph Stamm, «Determinanten der Länderwahl privater schweizerischer Entwicklungshilfe », Schweizerisches Jahrbuch für Entwicklungspolitik [Online], 23-2 | 2004, Online erschienen am: 10 Juni 2010, abgerufen am 08 September 2020. URL : http://journals.openedition.org/sjep/499 ; DOI : https://doi.org/10.4000/sjep.499 


\title{
Determinanten der Länderwahl privater schweizerischer Entwicklungshilfe
}

\author{
Christoph Stamm*
}

\section{Einführung}

Die aus privaten Beiträgen finanzierte jährliche „Auslandhilfe“ belief sich in der Schweiz in den letzten Jahren auf 280 bis 330 Millionen Franken ${ }^{1}$. Diese Beiträge setzen sich aus Entwicklungszusammenarbeit, humanitärer Hilfe und Hilfe an Transitionsländer zusammen. Die Gelder werden von den schweizerischen Hilfswerken und anderen Nichtregierungsorganisationen (NRO) beinahe ausschliesslich für bilaterale Projekte in Entwicklungs- und Transitionsländern eingesetzt. Im Gegensatz zur öffentlichen Hilfe wird die private Hilfe nicht von einer zentralisierten Stelle koordiniert und organisiert. Die private Hilfe ist dezentral und wird von einer Vielzahl kleiner und einigen grossen Hilfswerken und NRO geplant und durchgeführt ${ }^{2}$.

Es ist interessant zu fragen, ob sich aus der grossen Anzahl dezentral gefällter Entscheidungen der verschiedenen im Entwicklungsbereich tätigen privaten Organisationen zur Wahl von Projekten und deren geografischer Situierung ein kohärentes Gesamtbild ergibt. Wir gehen davon aus, dass die privaten Beiträge nicht nach dem Giesskannenprinzip gleichmässig auf die entsprechenden Länder verteilt werden, sondern dass die begrenzten Mittel nach bestimmten Prioritäten in die Länder des Südens und des Ostens fliessen. In dieser Analyse sollen demnach die tieferliegenden allgemeinen Determinanten bestimmt werden, welche die dezentral gefällte Länderwahl der privaten Hilfe beeinflussen.

Unsere Hypothese ist folgende: Die geografische Verteilung der privaten Hilfe der Schweiz wird durch zwei Kategorien von Faktoren beeinflusst. Die erste Kategorie besteht aus entwicklungsländerspezifischen, der Schweiz externen Faktoren. Dazu gehören der wirtschaftliche und soziale Entwicklungsstand eines Landes, seine humanitäre Lage und sein Platz in der internationalen Empfängergemeinschaft. Die zweite Kategorie besteht aus Schweiz-internen Faktoren. Dazu zählen die Verteilung der öffentlichen Entwicklungshilfe, die aus Entwicklungs- und Transitionsländern stammende Wohnbevölkerung oder die geografische Distanz der Empfängerländer zur Schweiz. Ein besonderes Augenmerk legen wir dabei auf die Beziehung zwischen der privaten Hilfe und der öffentlichen Entwicklungszusammenarbeit, da vieles darauf hindeutet, dass letztere einen beträchtlichen Einfluss auf die private Hilfe ausübt.

* Politologe.

1 Da nicht alle Hilfswerke und NRO Angaben über ihre Budgets für die Entwicklungszusammenarbeit machen, liegt der tatsächliche Gesamtbeitrag höher.

2 Zur Struktur und Rolle der schweizerischen NRO siehe den Beitrag von Gérard Pérroulaz in diesem Dossier: Die Rolle der NRO in der Entwicklungspolitik: Stärken und Grenzen, Legitimität und Kontrolle. 
Angesichts der oftmals auf individuelle Initiative durchgeführten Projekte nehmen wir ausserdem an, dass bei der geografischen Verteilung auch der Faktor ,individuelle Präferenzen“ eine beträchtliche Rolle spielt.

\section{Ursachenmodell}

Externe und länderspezifische Faktoren

(darunter "humanitäre Aspekte“)

Schweizspezifische Faktoren

(darunter "offentlichen Hilfe")

Individuelle Präferenzen

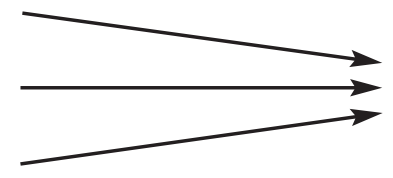

geografische Verteilung

der privaten Hilfsgelder

aus der Schweiz

Die Basis für diese quantitative Analyse bilden die privaten Beiträge aus der Schweiz für das Jahr 2001. Berücksichtigt werden 150 Entwicklungs- und Transitionsländer mit mindestens 200'000 Einwohnern. Im nächsten Teil werden wir uns einen Überblick über die geografische Verteilung verschaffen. Dann testen wir die verschiedenen Teilhypothesen, und zum Schluss wird ein geprüftes Gesamtmodell über die Determinanten der geografischen Verteilung privater Hilfe Auskunft geben.

\section{Geografische Verteilung und Konzentration}

\section{Hilfe nach Ländern}

Im Jahr 2001 flossen 330,6 Millionen Franken an privater Hilfe in Entwicklungsund Transitionsländer. Davon können 296 Millionen (ca. 90\%) einzelnen Ländern zugeordnet werden; 34,6 Millionen (ca. 10\%) flossen in länderübergreifende Projekte und können keinem bestimmten Land zugeordnet werden. 127 Länder profitierten von den privaten Geldflüssen aus der Schweiz, 65 Länder erhielten über eine Million Franken. Aus Tabelle 1 werden grosse Beitragsunterschiede ersichtlich. Sie zeigt die zwanzig wichtigsten Empfängerländer, welche gut 60 Prozent der zugeordneten privaten Hilfe bekommen ${ }^{3}$. Weiter ist erkennbar, dass sechs Länder zusammen mehr als ein Drittel aller Beiträge erhalten.

Um zu sehen, ob sich die Höhe der Beiträge mit der Grösse des Landes erklären lassen, lohnt es sich, die Beitragshöhe mit der Bevölkerungszahl in Beziehung zu setzen. Tabelle 2 zeigt daher die wichtigsten Empfängerländer unter Einbezug ihrer Bevölkerung. Mit der Darstellung der Pro-Kopf-Hilfe wird ersichtlich, wie viel finanzielle Hilfe die Bevölkerung und die einzelnen Personen erhalten. Die bevölkerungsreichsten Staaten gehören nun nicht mehr zu den Hauptempfängern. Neun Staaten (im Fettdruck in der Tabelle 1 und 2) finden in beiden Kategorien einen Platz unter den ersten zwanzig. Die grossen Unterschiede zwischen den Staaten bleiben aber auch mit dieser Kategorisierung bestehen.

3 Auf den Plätzen 21 bis 30 stehen die Länder Guatemala, Rumänien, Bangladesch, Kenia, Haiti, Kamerun, Burkina Faso, Vietnam, Tschad und Uganda. 
Trotz einem Geldfluss in 127 von 150 Entwicklungs- und Transitionsländern gibt es eine relativ hohe Konzentration der Beiträge in wenige Länder. Dies legt die Vermutung nahe, dass die privaten Hilfswerke bei ihrer Wahl der Empfängerländer Schwerpunkte setzen. Im Kapitel „Faktoren, welche die Länderwahl beeinflussen“ wollen wir uns an die Determinanten dieser Prioritätensetzung herantasten.

Tabelle 1: Die 20 wichtigsten Empfängerländer privater Hilfe 2001 (in absoluten Zahlen)

\begin{tabular}{lccc}
\hline Land & $\begin{array}{c}\text { Private Hilfe in } \\
\text { Tausend Franken }\end{array}$ & $\begin{array}{c}\text { Private Hilfe in } \\
\text { Prozent }\end{array}$ & $\begin{array}{c}\text { Private Hilfe in } \\
\text { Prozent kumuliert }\end{array}$ \\
\hline 1. Jugoslawien & 27200 & 9.2 & 9.2 \\
\hline 2. Indien & 21646 & 7.3 & 16.5 \\
\hline 3. Kambodscha & 19959 & 6.7 & 23.2 \\
\hline 4. Mosambik & 11005 & 3.7 & 27.0 \\
\hline 5. Sudan & 10659 & 3.6 & 30.6 \\
\hline 6. Brasilien & 8455 & 2.9 & 33.4 \\
\hline 7. Honduras & 7047 & 2.4 & 35.8 \\
\hline 8. Kolumbien & 6913 & 2.3 & 38.1 \\
\hline 9. Tansania & 6712 & 2.3 & 40.4 \\
\hline 10. Nicaragua & 5897 & 2.0 & 42.4 \\
\hline 11. Äthiopien & 5885 & 2.0 & 44.4 \\
\hline 12. Albanien & 5825 & 2.0 & 46.4 \\
\hline 13. El Salvador & 5661 & 1.9 & 48.3 \\
\hline 14. Bolivien & 5562 & 1.9 & 50.1 \\
\hline 15. Peru & 5472 & 1.8 & 52.0 \\
\hline 16. Philippinen & 5169 & 1.7 & 53.7 \\
\hline 17. Afghanistan & 5091 & 1.7 & 55.5 \\
\hline 18. Türkei & 5036 & 1.7 & 57.2 \\
\hline 19. Bosnien-Herzegowina & 4834 & 1.6 & 58.8 \\
\hline 20. Dem. Rep. Kongo & 4458 & 1.5 & 60.3 \\
\hline Übrige Länder & 117492 & 39.7 & 100.0 \\
\hline Total & $\mathbf{2 9 5 9 7 8}$ & $\mathbf{1 0 0 . 0}$ & \\
\hline Pro Land (zugeordnete Hilfe) & 1973 & & \\
\hline Pro Land (gesamte bilaterale Hilfe) & 2204 & & \\
\hline
\end{tabular}

Tabelle 2: Die 20 wichtigsten Empfängerländer privater Hilfe (pro Kopf der Bevölkerung)

\begin{tabular}{lclc}
\hline Land & Beitrag pro Kopf in Rappen & \multicolumn{1}{l}{ Land } & Beitrag pro Kopf in Rappen \\
\hline 1. Jugoslawien & 257 & 11. Bolivien & 67 \\
\hline 2. Albanien & 188 & 12. Mosambik & 60 \\
\hline 3. Kambodscha & 152 & 13. Palästina (von Israel besetzte Gebiete) & 54 \\
\hline 4. Bosnien-Herzegowina & 124 & 14. Libanon & 51 \\
\hline 5. Nicaragua & 116 & 15. Haiti & 48 \\
\hline 6. Honduras & 110 & 16. Armenien & 47 \\
\hline 7. Gabun & 97 & 17. Swasiland & 45 \\
\hline 8. El Salvador & 90 & 18. Tschad & 42 \\
\hline 9. Bhutan & 71 & 19. Eritrea & 39 \\
\hline 10. Mauretanien & 71 & 20. Guatemala & 38 \\
\hline pro Kopf der gesamten Bevölkerung (zugeordnete Beiträge) & 5.69 \\
\hline pro Kopf der gesamten Bevölkerung (aller bilateralen Beiträge) & 6.36 \\
\hline
\end{tabular}




\section{Hilfe nach Kontinenten}

Grafik 1 zeigt die geografische Verteilung der privaten Spenden nach Kontinenten. Afrika und Asien erhalten ein knappes Drittel der Gelder, die Entwicklungsländer Amerikas ein gutes Fünftel, nach Europa fliesst ein gutes Sechstel aller Beiträge. Das Verhältnis des Kontinents, der am wenigsten erhält (Europa) zu dem, der am meisten erhält (Afrika), beträgt 1:2. Setzen wir nun die Beiträge in Beziehung zu den Bevölkerungszahlen, so zeigt sich ein ganz anderes Bild (Grafik 2). Die Einwohner der europäischen, afrikanischen und amerikanischen Entwicklungs- und Transitionsländer erhalten zwischen 12 und 13 Rappen pro Person. Die Bevölkerungen der asiatischen Länder erhalten nur 2,6 Rappen pro Person. Dies zeigt, dass das Gewicht Asiens in der privaten Hilfe sehr viel geringer ist, als das Gewicht der anderen Kontinente. Es ist jetzt Europa, das am meisten von der privaten Hilfe profitiert. Das Verhältnis zwischen Asien und Europa beträgt 1:5.

\section{Grafik 1: Beiträge nach Kontinenten (in Prozenten)}

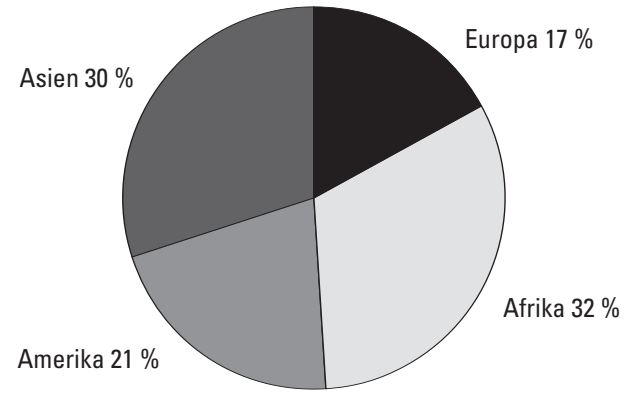

Grafik 2: Beiträge pro Kopf der Bevölkerung (in Rappen)

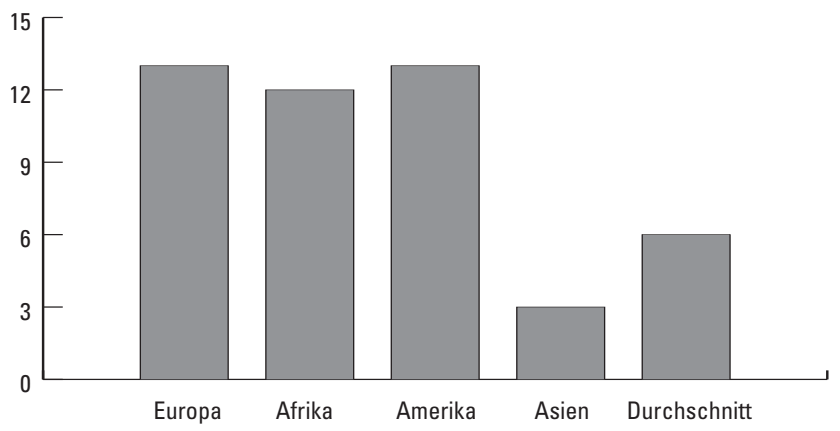

\section{Hilfe nach Einkommenskategorien}

Eine Übersicht der Verteilung der Beiträge nach dem Pro-Kopf-Einkommen der Länder gibt erste Hinweise auf eine mögliche Prioritätensetzung der privaten Hilfswerke. Wir stützen uns dabei auf die Einkommenskategorien der OECD aus dem Jahr 20014. Die hier der Anschaulichkeit und der Bevölkerungsgrösse

4 DAC-Liste der Hilfeempängerländer (1. Januar 2001) im Jahrbuch 2004, Nr. 1. 
wegen ausgegliederten Länder Indien und China gehören zur Kategorie der Länder mit tiefem Einkommen ${ }^{5}$.

Grafik 3 zeigt, dass sich die beiden Kategorien „tiefes Einkommen“ und „mittleres Einkommen untere Stufe" über 80 Prozent der Hilfe teilen. Grafik 4 zeigt die Pro-Kopf-Beiträge für jede Einkommenskategorie. Die Bevölkerungen der Länder in der Kategorie ,,mittleres Einkommen untere Stufe“ erhalten am meisten von der privaten Hilfe aus der Schweiz. Hätte man China und Indien in der Kategorie der ihnen entsprechenden Kategorie der „tiefen Einkommen“ aufgeführt, so wären die Pro-Kopf-Beiträge dieser Kategorie auf 4,7 Rappen gesunken, also praktisch auf die Höhe der Kategorie ,mittleres Einkommen obere Stufe". Dieser Überblick deutet an, dass sich die private Hilfe weder auf die ärmsten, noch auf die reichsten Entwicklungsländer fokussiert. Die Konzentration liegt vielmehr auf den Ländern im mittleren Einkommensbereich.

\section{Grafik 3: Aufteilung der Gelder nach Einkommenskategorie}

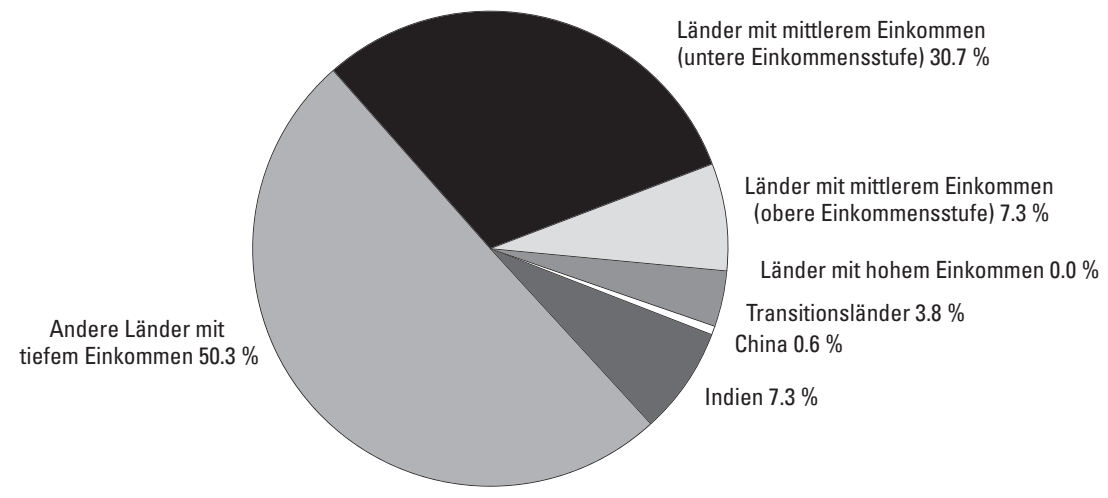

Grafik 4: Aufteilung pro Kopf und pro Einkommenskategorie (in Rappen)

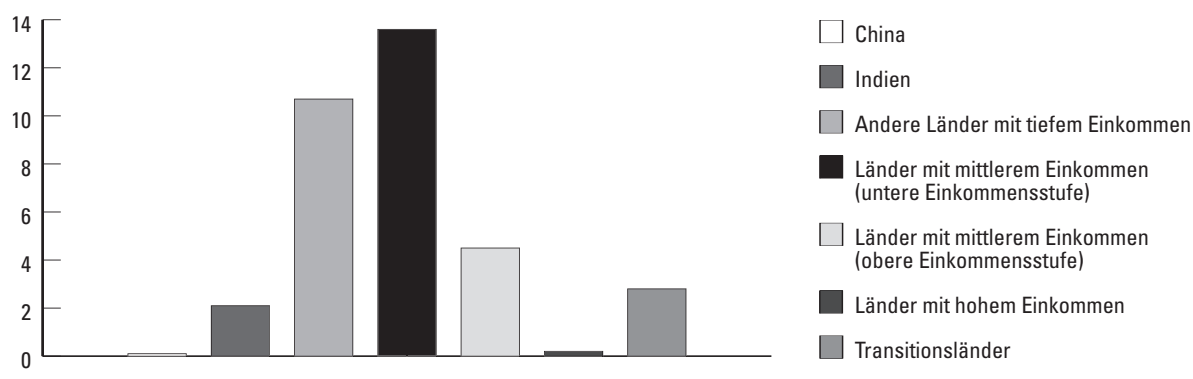

5 Indien und China können damit auch mit den Grafiken 1 und 2 (Hilfe nach Kontinenten) verglichen werden. 


\section{Synthese}

Wir haben gesehen, dass die Gelder in eine grosse Anzahl Länder fliessen, dass die Beiträge pro Land sehr grosse Unterschiede aufweisen, und dass sich 50 Prozent der Hilfe auf vierzehn Länder konzentriert. Weiter wurde ersichtlich, dass der bevölkerungsreiche asiatische Kontinent relativ zur Bevölkerung klar weniger Hilfe erhält als die anderen Kontinente. Schliesslich konnten wir eine Konzentration der Hilfe auf Entwicklungsländer mit mittlerem Einkommen feststellen. Im folgenden Teil werden wir unsere Grundhypothesen zur Aufteilung der privaten Hilfe erläutern und testen.

\section{Faktoren, welche die Länderwahl beeinflussen}

\section{Externe und länderspezifische Faktoren}

\section{$\square$ Entwicklungsstand eines Landes}

Bei dieser Hypothese wird davon ausgegangen, dass Charakteristika der Entwicklungsländer die geografische Verteilung der Hilfe bestimmen. Zu nennen wäre da in erster Linie der Entwicklungsstand der entsprechenden Länder. Eine kohärente Entwicklungszusammenarbeit mit der Priorität der Armutsbekämpfung würde die ärmsten Länder am meisten unterstützen. Die Teilhypothese ist daher folgende: Je ,weniger entwickelt“ ein Land, desto grösser die privaten Beiträge aus der Schweiz. Für die Bestimmung des Entwicklungsstandes verwenden wir den „Human Development Index“ der Vereinten Nationen, welcher das „Wohlbefinden“ der einzelnen Länder misst. Die oben aufgezeigte Verteilung nach Einkommenskategorien liess es erahnen: Die lineare Regressionsanalyse zeigt keinen signifikanten Zusammenhang zwischen dem Stand der menschlichen Entwicklung eines Landes und dessen privater Unterstützung aus der Schweiz ${ }^{6}$. Die Hypothese muss daher verworfen werden.

\section{$\square$ Soziale Notlagen und humanitäre Aspekte}

Die Hilfswerke sind auf individuelle Spenden angewiesen und richten daher ihre Entwicklungszusammenarbeit zumindest teilweise auf die Präferenzen der Spender aus. Diese Teilhypothese geht davon aus, dass soziale Notlagen, verursacht durch Kriege, Naturkatastrophen und Ernteausfälle den potenziellen Spender zu einer Spende bewegen ${ }^{7}$. Je grösser die menschlichen Katastrophen in einem Land, desto mehr Beiträge fliessen an den entsprechenden Ort. Tatsächlich liegt der Anteil der humanitären Hilfe bei der privaten Hilfe zwischen einem Viertel und einem Drittel und damit höher als bei der öffentlichen Hilfe.

Als Indikatoren für die Ursachenanalyse wählten wir für die Messung der Schwere der kriegerischen Ereignisse die Kriegsopferzahlen des schwedischen

6 Siehe auch Annex. Auch die Variablen „Demokratie/Bürgerrechte“ (Indikator von Freedom House) und „Good Governance“ (Indikator der Weltbank) haben keinen Einfluss auf die Länderwahl.

7 Die nationalen Sammeltage der Glückskette nach Katastrophen (1998 für mittelamerikanische Opfer des Hurrikans Mitch, 1999 für türkische Erdbebenopfer und für jugoslawische Kriegsopfer) brachten Spenden von bis zu 50 Millionen Franken ein, was bis zu ein Sechstel des jährlichen Spendenaufkommens ausmacht. 
Konfliktforschungsinstitutes SIPRI der Jahre 1997 bis 2001. Für die Messung der Schwere von Naturkatastrophen wählten wir die Daten über Opferzahlen des Rückversicherungskonzerns SwissRe der Jahre 1998 bis 2001. Als allgemeinen Indikator für die humanitäre Situation der entsprechenden Landesbevölkerungen wählten wir die Zahl der unterernährten Menschen der Periode 1999/2001 aus den Daten der FAO ${ }^{8}$.

Die Korrelationsanalyse zeigt einen statistisch signifikanten positiven Zusammenhang zwischen den „Katastrophen-Indikatoren“ und der geografischen Verteilung der Hilfe auf. Dabei wirkt sich vor allem die Variable „Unterernährung" auf die Verteilung aus; die Variable „Naturkatastrophe“ hat dagegen kaum einen Einfluss auf die Verteilung. Kumuliert man in einer linearen Regressionsanalyse die Variablen „Unterernährung“ und „Kriege“, so können diese knapp ein Viertel $\left(r^{2}=0.24\right)$ der geografischen Verteilung privater Hilfe erklären.

Die Teilhypothese, dass die private Hilfe in einem bestimmten Mass (aber vielleicht weniger, als oftmals vermutet) von Katastrophen in Entwicklungsländern abhängig ist, konnte erhärtet werden. Es stellt sich aber die Frage, ob vielleicht nicht so sehr die Katastrophen an sich, sondern viel eher deren Mediatisierung in der Schweiz die privaten Spenden beeinflusst ${ }^{9}$.

\section{$\square$ Die „Modeländer“ der internationalen Hilfe}

In dieser Teilhypothese gehen wir davon aus, dass es Entwicklungsländer gibt, welche einerseits sehr offen für ausländische Hilfe sind und andererseits richtiggehende Lieblinge der internationalen Hilfe darstellen. Nach dem Motto „wer bekommt, dem geben auch die Schweizer Hilfswerke“ wird vermutet, dass die geografische Verteilung privater Hilfe von den Beiträgen der weltweiten Gebergemeinschaft abhängt. Als Indikator verwenden wir dabei die weltweit gesamten Hilfeleistungen, welche ein entsprechendes Entwicklungsland erhält ${ }^{10}$. Das Resultat der Analyse ist erstaunlich. Der Zusammenhang der Variablen ist statistisch signifikant und positiv. Die Hypothese wird erhärtet, da 26 Prozent der Varianz durch die Regressionsgleichung erklärt werden ${ }^{11}$.

\section{Schweizspezifische Faktoren}

Wir gehen bei dieser Hypothese davon aus, dass die private Hilfe durch ihre Einbettung in den schweizerischen Kontext durch diesen auch stark beeinflusst wird. Diese Beeinflussung wirkt sich auch auf die geografische Verteilung aus. Es sind aber weder die geografische Distanz der entsprechenden Länder zur Schweiz, noch die sprachlich-kulturelle Distanz, welche einen Einfluss auf die Verteilung haben. Wir vermuten vielmehr, dass die Immigration, das heisst die

Zur Konstruktion der Indikatoren und zur Regression siehe auch Annex.

9 So flossen zum Beispiel nach dem stark mediatisierten Kosovokonflikt in Jugoslawien sehr viele Spenden in diese Region. Wenig Geld fliesst dagegen in das Gebiet des ,vergessenen“ Tschetschenienkrieges.

10 Zur Konstruktion der Indikatoren und zur Regression siehe auch Annex.

11 Der Zusammenhang wird auch durch einen Vergleich zwischen den 20 wichtigsten Empfängerländern der weltweit gesamten Hilfe und den wichtigsten privaten Empfängerländern aus Tabelle 1 deutlich. Die acht Länder Äthiopien, Bolivien, Honduras, Indien, Jugoslawien, Mosambik, Nicaragua und Tansania befinden sich auf beiden Listen. 
Anzahl in der Schweiz wohnenden Personen aus den entsprechenden Entwicklungsländern sowie die öffentliche Hilfe des Bundes die Länderwahl der privaten Hilfe beeinflussen.

\section{$\square$ Eingewanderte Wohnbevölkerung aus Entwicklungsländern}

Die erste Teilhypothese lautet folgendermassen: Je mehr Personen aus dem entsprechenden Entwicklungs- oder Transitionsland in der Schweiz wohnen, desto mehr finanzielle Hilfe erhält dieses Land. Der Einfluss könnte auch in die andere Richtung gehen: Je mehr finanzielle Hilfe ein Land erhält, desto mehr Personen wandern aus dem entsprechenden Land in die Schweiz ein. Aus nachfolgenden Gründen nehmen wir aber an, dass die Richtung des Einflusses der erstgenannten Hypothese entspricht. Die eingewanderte Wohnbevölkerung in der Schweiz weckt das Interesse der Schweizer Bevölkerung für die entsprechenden Länder. Die Immigranten und die Schweizer Bevölkerung lernen sich gegenseitig kennen. Durch die so entstandene emotionale Nähe ist die Schweizer Bevölkerung bereit, etwas für die Verbesserung der Lebensbedingungen in den entsprechenden Ländern beizutragen. Gleichzeitig können sich die Hilfswerke auf das Wissen und die Erfahrung der eingewanderten Personen stützen und mit Projekten in deren Ursprungsländern Synergien nutzen. Zudem gründen die in der Schweiz eingewanderten Personen Unterstützungsvereine für ihre Herkunftsregion und finanzieren Projekte zur Unterstützung der heimatlichen Bevölkerung.

Als Indikator für die aus Entwicklungs- und Transitionsländern eingewanderte Wohnbevölkerung verwenden wir die Zahlen der Volkszählung 2000 des Bundesamtes für Statistik ${ }^{12}$.

In der linearen Regression setzen wir die Variable „eingewanderte Wohnbevölkerung der einzelnen Länder" als unabhängige Variable, die schweizerische private Hilfe in die einzelnen Länder als abhängige Variable ein. Die lineare Regression ist statistisch signifikant und die Beziehung ist stark. Die eingewanderte Wohnbevölkerung aus südlichen und östlichen Ländern erklärt 28 Prozent der geografischen Verteilung der privaten Hilfe ${ }^{13}$.

\section{$\square$ Beziehung Hilfswerke - Bund (DEZA)}

Vieles deutet darauf hin, dass die geografische Verteilung der privaten Hilfsgelder in Entwicklungs- und Transitionsländer der Verteilung der öffentlichen Gelder ähnlich ist. Die Hauptthese dieser Arbeit lautet deshalb folgendermassen: Die Länderaufteilungen der privaten und der öffentlichen Hilfe sind sich sehr ähnlich und beeinflussen sich gegenseitig. Das bedeutet, dass die privaten und die öffentlichen Entwicklungsgelder zu einem beträchtlichen Teil in die gleichen Länder fliessen - Bund und private Hilfswerke haben dieselben Schwerpunktländer. Der Einfluss der öffentlichen Entwicklungsarbeit auf die private Entwicklungszusammenarbeit ist aber grösser als umgekehrt. Folgende Gründe sprechen für diese Hypothese :

12 Zur Konstruktion der Indikatoren und zur Regression siehe auch Annex.

13 Sieben Länder finden sich gleichzeitig auf der Liste der 20 wichtigsten Empfängerländer privater Hilfe und auf der Liste der 20 wichtigsten aus Entwicklungs- und Transitionsländern eingewanderten Nationen; siehe Annex, Tabelle 5. 
In der Schweiz gingen in den 50er Jahren private Initiativen und Aktivitäten von Missionswerken der öffentlichen bilateralen Entwicklungszusammenarbeit voraus. Dies ist wohl hauptsächlich darauf zurückzuführen, dass die Schweiz keine Kolonien besass. 1961 wurde dann vom Bund ein Dienst für technische Zusammenarbeit geschaffen, um öffentliche bilaterale Projekte durchzuführen. Eine Koordination mit den privaten Hilfswerken war ausdrücklich vorgesehen. Der Bund profitierte von den Erfahrungen der Hilfswerke; die letzteren wurden zu Ausführenden bilateraler Projekte des Bundes. Für seine bilaterale Entwicklungszusammenarbeit wählte der Bund dabei teilweise Länder aus, in denen die privaten Institutionen bereits aktiv waren ${ }^{14}$.

Die Zusammenarbeit des Bundes mit den Hilfswerken wurde auch gesetzlich festgeschrieben: „Der Bundesrat kann Bestrebungen privater Institutionen, die den Grundsätzen und Zielen dieses Gesetzes entsprechen, mit den ihm zur Verfügung stehenden Mitteln unterstützen. Die Institutionen haben eine angemessene Eigenleistung zu erbringen." 15

Die Botschaft über die Weiterführung der Entwicklungshilfe von 2003 zeugt vom engen Verhältnis des Bundes mit den Hilfswerken: „Seit ihren Ursprüngen zeichnet sich die schweizerische Entwicklungszusammenarbeit durch eine intensive Zusammenarbeit mit schweizerischen Nichtregierungsorganisationen aus, sowohl im operationellen wie im entwicklungspolitischen Bereich“. ${ }^{16}$ Und auch die Botschaft über die Weiterführung der humanitären Hilfe spricht von einer engen Partnerschaft: „Die im humanitären Bereich aktiven Schweizer Hilfswerke spielen eine wichtige Rolle bei der Durchführung von Projekten. Sie teilen ihre Erfahrungen mit der DEZA, wodurch Synergien möglich werden" "17.

Die schweizerischen NRO führen Projekte und Programme der DEZA durch (so genannte Mandate oder Regieaufträge). Zudem leistet die DEZA Beiträge an Entwicklungsprojekte, welche von schweizerischen Hilfswerken in eigener Verantwortung durchgeführt werden (mehrjährige Programmbeiträge an 13 grössere NRO). Insgesamt wickelt die DEZA 25 bis 30 Prozent ihrer bilateralen Hilfe über schweizerische NRO ab. Der Anteil der öffentlichen Beiträge, gemessen an den gesamten Aktivitäten der privaten Organisationen, beläuft sich auf 35 bis 40 Prozent $^{18}$.

Seit den 90er Jahren des letzten Jahrhunderts werden die Mandate der DEZA in der Regel öffentlich ausgeschrieben, und auch nichtschweizerische NRO und die Privatwirtschaft können sich dafür bewerben. Bewusst wird damit der Konkurrenzdruck unter den Hilfswerken erhöht ${ }^{19}$.

14 Zum Beispiel entstanden in Nepal erste Projekte des Hilfswerks Helvetas im Jahr 1956. Das Land wurde dann im Jahr 1962 zu einem ,Schwerpunktland“ der öffentlichen Entwicklungszusammenarbeit. Im Tschad unterstützte der Bund ab 1964 Aktivitäten von Schweizer Missionaren und auch dieses Land wurde zu einem Schwerpunktland der öffentlichen Entwicklungszusammenarbeit.

15 Bundesgesetz über die internationale Entwicklungszusammenarbeit und humanitäre Hilfe von 1976.

16 Bundesrat, Botschaft über die Weiterführung der technischen Zusammenarbeit und der Finanzhilfe zu Gunsten von Entwicklungsländern, vom 28. Mai 2003, S. 2668.

17 Bundesrat, Botschaft über die Weiterführung der internationalen humanitären Hilfe der Eidgenossenschaft, vom 14. November 2001, S. 2244.

18 Bundesrat, Botschaft über die Weiterführung der technischen Zusammenarbeit und der Finanzhilfe zu Gunsten von Entwicklungsländern, op. cit., S. 2669.

19 So zum Beispiel bei der so genannten „Osthilfe“: „,...werden die Aufträge der Ostzusammenarbeit im Wettbewerb vergeben.“ Der Bund trägt damit „zur Stärkung des Wettbewerbs zwischen den Anbietern 
Diese enge Zusammenarbeit deutet auf eine gleiche Länderwahl von Bund und Hilfswerken hin. $\mathrm{Zu}$ Beginn der öffentlichen Entwicklungszusammenarbeit beeinflussten die privaten Institutionen die Länderwahl des Bundes. Mit der Zeit wurde jedoch die bilaterale Zusammenarbeit des Bundes immer bedeutender und deren Beiträge überflügelten diejenigen der privaten Hilfe. Damit wurde der Bund in seiner Länderwahl selbstständiger. Für diejenigen Hilfswerke, welche mit dem Bund zusammenarbeiten, ist es aus Synergiegründen nahe liegend, die privat finanzierten Projekte in denselben Ländern durchzuführen, in denen sie auch die Regieaufträge des Bundes ausführen. Die öffentliche Ausschreibung der Bundesaufträge verstärkt diesen Trend. Ein Hilfswerk erhält einen öffentlichen Auftrag, sofern es gegenüber anderen Hilfswerken (und gegenüber den bundeseigenen Leistungen) einen komparativen Vorteil hat ${ }^{20}$. Ein solcher Auftrag ist leichter erhältlich, wenn das kandidierende Hilfswerk im entsprechenden Land bereits über Erfahrung verfügt. Damit steigt das Interesse der Hilfswerke, in den aktuellen Schwerpunktländern der DEZA mit eigenen Projekten aktiv zu sein und die zukünftigen Schwerpunktländer der DEZA zu erahnen. Daher denken wir, dass der Bund die Länderwahl der privaten Institutionen heute stärker beeinflusst, als umgekehrt.

Kommen wir nun zur empirischen Analyse. Als Indikator verwenden wir die gesamten öffentlichen Leistungen an Entwicklungs- und Transitionsländer aus dem Jahr 2001. Die lineare Regression zeigt einen sehr starken positiven Zusammenhang zwischen öffentlicher und privater Hilfe $\left(\mathrm{R}=0.71, \mathrm{r}^{2}=0.51\right)^{21}$. Demnach können 51 Prozent der Verteilung der privaten Hilfe durch die geografische Verteilung der öffentlichen Hilfe erklärt werden.

Der starke Zusammenhang wird auch durch einen Vergleich zwischen den zwanzig wichtigsten Empfängerländern der öffentlichen Hilfe und den wichtigsten privaten Empfängerländern aus Tabelle 1 deutlich. Acht Länder finden sich auf beiden Listen ${ }^{22}$. Die Analyse wurde aber eventuell dadurch verzerrt, dass der Bund einen beträchtlichen Teil der bilateralen Hilfe länderübergreifend einsetzt und damit keinem bestimmten Land zuweist. Während bei der privaten Hilfe nur 10 Prozent nicht zugeordnet werden können, sind es bei der öffentlichen Hilfe 35 Prozent. Wegen dieser „Unpräzision“ von Seiten des Bundes könnte der tatsächliche Zusammenhang zwischen privater und öffentlicher Hilfe um einiges grösser oder geringer sein.

bei." Explizit werden auch potenzielle ausländische Bewerber erwähnt (Bundesrat, Botschaft über die Weiterführung der verstärkten Zusammenarbeit mit Osteuropa und den Staaten der GUS, vom 19. August 1998, S. 35).

20 Ähnliches gilt für die humanitäre Hilfe: Der Bund überträgt den Hilfswerken Aufgaben, ,,wenn ein Hilfswerk einen komparativen Vorteil hat" (Bundesrat, Botschaft über die Weiterführung der internationalen humanitären Hilfe der Eidgenossenschaft, vom 14. November 2001, S. 2244).

21 Siehe auch Grafik 5.

22 Siehe Tabelle 4 im Annex. 


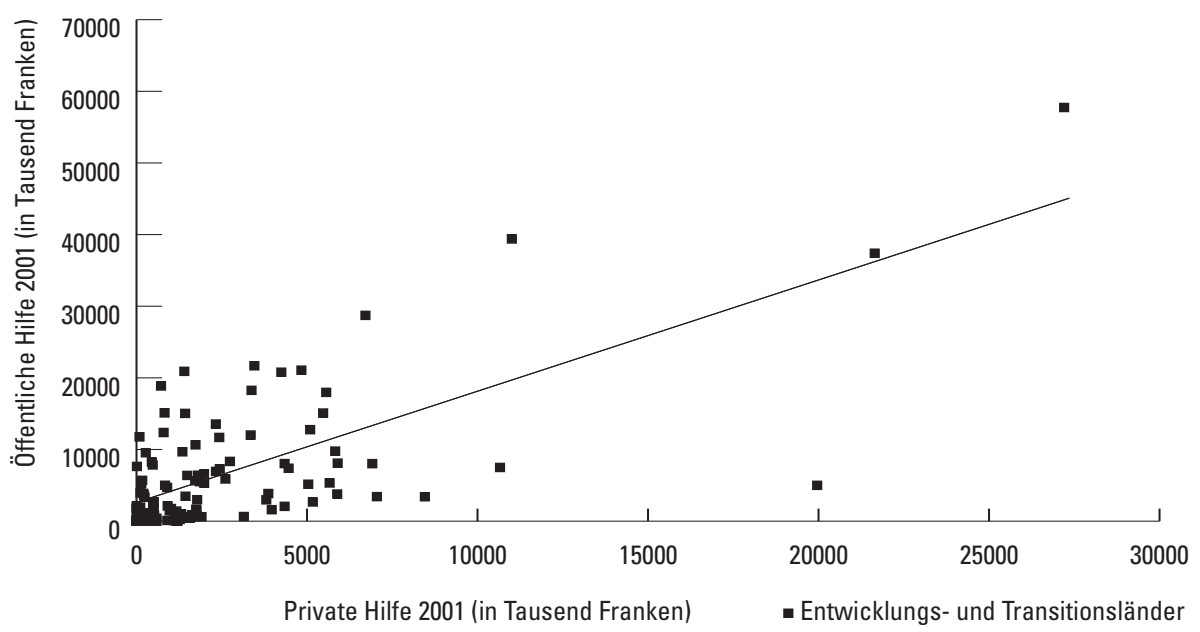

\section{Individuelle Präferenzen}

Gerade weil die private Hilfe dezentral und nicht durch eine Institution koordiniert ist, spielen bei der geografischen Verteilung der Hilfe die individuellen Präferenzen eine nicht unbedeutende Rolle. Das Element wird dadurch verstärkt, dass die Schweiz ein kleines Land und der Gesamtbetrag der privaten Hilfe dementsprechend bescheiden ist. Daher können individuelle Initiativen sowie Interessen und Opportunitäten eines Einzelnen (oder einer kleinen Gruppe) einen beträchtlichen Einfluss auf die geografische Verteilung haben. Zwei besonders markante Beispiele mögen dies illustrieren: Im Jahr 2001 erhielt Kambodscha knapp 20 Millionen Franken an privaten Beiträgen. Dies entspricht einem Anteil von 6,7 Prozent der gesamten Hilfe; das Land steht damit an dritter Stelle der Empfängerländer. Schauen wir uns genauer an, wie sich diese Hilfe zusammensetzt, so sehen wir, dass allein die Beiträge an die Stiftung Kinderspital Kantha Bopha über 16 Millionen Franken ausmachen. Würde dieses sehr stark an eine Einzelperson gebundene Projekt nicht existieren oder in einem anderen Land (warum nicht z.B. in Laos) stattfinden, so würde Kambodscha nur an sechsundzwanzigster Stelle stehen.

Als zweites Beispiel nehmen wir Albanien. Das Land stand bei der privaten ProKopf-Hilfe an zweiter Stelle. Über 60 Prozent der Beiträge an Albanien stammten vom Verein Operazione Ticinese Aiuti Albania (O.T.A.A.). Dieser wurde mit dem einzigen Ziel gegründet, die (katholische) Bevölkerung der Diözese Rrèshen zu unterstützen. Ohne diese Initiative einer kleinen Gruppe würde Albanien bei der Pro-Kopf-Hilfe erst an neunter Stelle stehen.

Diese Beispiele machen deutlich, dass ein einzelnes Projekt oder eine individuelle Initiative in einem ,zufällig“ gewählten Land den Schlüssel der geografischen Verteilung sehr stark beeinflussen kann. Es ist jedoch schwierig, den Einfluss der individuellen Präferenzen zu quantifizieren. 


\section{Synthese}

Schliessen wir jetzt die wichtigsten unabhängigen Variablen in ein Gesamtmodell ein. Der gewichtigste Faktor ist die „öffentliche Hilfe“. Fügt man die Variable ,gesamte Hilfeleistungen der internationalen Gebergemeinschaft" hinzu, so trägt diese nur sehr wenig Zusätzliches zur Erklärung der abhängigen Variable ,private Hilfe“ bei. Daraus schliessen wir, dass die öffentliche Hilfe ihrerseits relativ stark der Logik der ,,internationalen Modeländer“ folgt.

Als zweitwichtigste unabhängige Variable fungiert die ,eingewanderte Wohnbevölkerung“. Ihr Gewicht beträgt 58 Prozent der Variable „öffentliche Hilfe“. Als dritten und letzten unabhängigen Faktor schliessen wir die Variable „Unterernährung" in unsere multiple Regression ein. Ihr Gewicht ist noch etwas schwächer und beträgt 53 Prozent der Variable „öffentliche Hilfe“.

Das Schlussmodell mit diesen drei unabhängigen Variablen erklärt 59 Prozent der abhängigen Variable ,private Hilfe“ $(\mathrm{r} 2=0.59)^{23}$. Damit bleiben 41 Prozent der Varianz unerklärt. Diese 41 Prozent setzen sich aus uns unbekannten Faktoren und aus den ,individuellen Präferenzen“ zusammen.

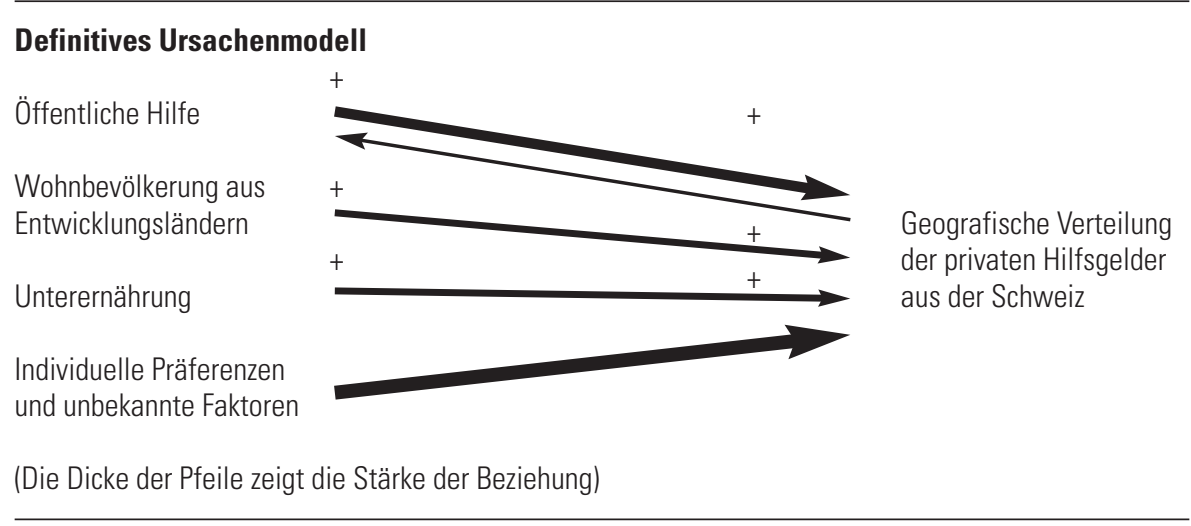

\section{Schlussfolgerung}

Wir haben gesehen, dass für die geografische Verteilung der privaten Hilfe in erster Linie interne Faktoren eine Rolle spielen. Mit Abstand an erster Stelle steht dabei das Gewicht der öffentlichen Hilfe. Die private und die öffentliche Hilfe sind, was die geografische Ausrichtung betrifft, nicht komplementär. Vielmehr teilen sie sich eine bedeutende Zahl von „Schwerpunkländern“. Bei dieser Ähnlichkeit stellt sich natürlich die Frage nach der Verteilungslogik der öffentlichen Hilfe. Welche Determinanten bestimmen denn die Länderwahl der öffentlichen Hilfe? Dieser Frage müsste in einer anderen Analyse nachgegangen werden.

Da die Höhe der privaten und öffentlichen Beiträge beträchtlichen jährlichen Schwankungen unterworfen ist, würde es Sinn machen, die beiden Variablen über mehrere Jahre hinweg zu vergleichen.

23 Siehe auch Annex. 
Die ,individuellen Präferenzen“ sind aus oben genannten Gründen wohl der zweitwichtigste Faktor bei der geografischen Ausrichtung privater Hilfe. Diesem Phänomen ist jedoch mit unserer quantitativen Analyse nicht beizukommen. Mit einer qualitativen Nachforschung könnte da ein besserer Einblick gewonnen werden.

Die aus Entwicklungsländern stammende Wohnbevölkerung stellt einen weiteren, jedoch weniger wichtigen internen Faktor für die Länderwahl dar.

Bei den äusseren Faktoren fällt einzig die Variable „Unterernährung“ ins Gewicht. Eine prekäre humanitäre Situation in einem Land hat demnach einen positiven Einfluss auf die Höhe der privaten Beiträge aus der Schweiz.

Erstaunlicherweise hat der Entwicklungsstand eines bestimmten Landes keinen Einfluss auf die Höhe der Hilfsleistungen; diese Hypothese musste demnach verworfen werden. Die private Hilfe ist nicht auf die am wenigsten entwickelten Länder ausgerichtet. Geht man davon aus, dass Entwicklungshilfe in erster Linie den ärmsten Ländern zugute kommen soll, so muss die private Hilfe als unkohärent bezeichnet werden.

Weiter ist der Einfluss von Kriegen und Naturkatastrophen relativ gering. In diesem Zusammenhang bleibt aber die Rolle der Medien zu untersuchen. Es müsste analysiert werden, inwieweit eine starke Mediatisierung einzelner Katastrophen und Kriege die Geldflüsse der privaten Hilfe in die entsprechenden Länder leiten.

Die Antwort auf unsere Frage nach der Logik der Länderwahl kann angesichts der Resultate dieser Arbeit auf folgenden Punkt gebracht werden: Die geografische Verteilung privater Hilfe aus der Schweiz hat sehr viel mit der Schweiz und sehr wenig mit den Entwicklungs- und Transitionsländern zu tun.

\section{QUELLEN}

Allan Pierre, „La complexité, le hasard et l'individu dans la théorie politique internationale“ in Michel Girard (sous la direction de), Individu et politique internationale, Economica, Paris, 1994.

FAO, The State of Food Insecurity in the World 2003, Rom, 2003.

Gerster Richard, Nord-Süd-Politik: abschreiben oder investieren? Perspektiven der schweizerischen Entwicklungszusammenarbeit, Orell Füssli Verlag, Zürich, 1995.

Grüninger Beat, "Unsere tägliche Katastrophe gib uns heute..., in Mosquito Nr. 4, Zürich, Juni 1997.

Landolf Daniel, Fankhauser Michael, Gekaufte Entwicklungshilfe? Die (Un)Abhängigkeit der Nichtregierungsorganisationen von Geld und Einfluss der staatlichen Entwicklungspolitik, Edition Soziothek, Köniz, 1996.

Le Monde diplomatique, L'Atlas du Monde diplomatique, „La diffusion des langues multi-étatiques“, Paris, Januar 2003.

PNUD, Rapport mondial sur le développement humain 2002, Editions De Boeck Université, Brüssel, 2002.

Bundesamt für Statistik, Zahlen der Volkszählung 2000 zur ausländischen Wohnbevölkerung, Neuenburg, 2002.

Bundesrat, Botschaft über die Weiterführung der verstärkten Zusammenarbeit mit Osteuropa und den Staaten der GUS, vom 19. August 1998.

Bundesrat, Botschaft über die Weiterführung der technischen Zusammenarbeit und der Finanzhilfe zugunsten von Entwicklungsländern, vom 7. Dezember 1998.

Bundesrat, Botschaft über die Weiterführung der internationalen humanitären Hilfe der Eidgenossenschaft, vom 14. November 2001. 
Bundesrat, Botschaft über die Weiterführung der technischen Zusammenarbeit und der Finanzhilfe zu Gunsten von Entwicklungsländern, vom 28. Mai 2003.

DEZA, Jahresbericht 2001 - Internationale Zusammenarbeit der Schweiz, DEZA, Bern, 2002.

DEZA, Aide de la Suisse aux pays en développement et aux pays en transition - statistiques 2001, DEZA, Bern, 2003.

DEZA, Statistischer Dienst, Bern.

Freedom House, Freedom in the World 2000-2001, <www.freedomhouse.org/research/freeworld/2001/ index.htm>.

Glückskette, Jahresberichte der Glückskette, Jahre 1998-2001, Bern.

OECD, Statistical Annex of the 2003 Development Cooperation Report, Paris, Januar 2004.

Schweizerische Rückversicherungsgesellschaft (Hg.), sigma Natur- und Man made-Katastrophen, Jahre 1998-2001, Zürich.

SIPRI, FIRST (Facts on International Relations and Security Trends), Major Armed Conflicts 1997-2001, $<$ http://first.sipri.org >.

World Bank, GRICS : Governance Research Indicator Country Snapshot - Composite Governance Indicator 2000, <http://info.worldbank.org/governance/kkz2002/govmap.asp>.

\section{INTERNET-ADRESSEN}

Operazione Ticinese Aiuti Albania: <www.otaa.ch>.

Stiftung Kantha Bopha: <www.beat-richner.ch>. 


\section{Annex}

\section{A) Variablen}

1. Private Hilfe aus der Schweiz in Entwicklungs- und Transitionsländer $\rightarrow$ Abhängige Variable "Private Hilfe"

Zur Operationalisierung des Konzeptes verwenden wir die aufgrund einer Umfrage erhobenen Zahlen aus dem Jahr 2001 über die privaten Gelder, welche von Stiftungen, Vereinen, Hilfswerken oder anderen Organisationen aus der Schweiz den Partnern in Entwicklungs- und Transitionsländern auf gemeinnütziger Basis für Aktionen der Entwicklungszusammenarbeit und der humanitären Hilfe zur Verfügung gestellt werden ${ }^{24}$.

\section{Abhängige Variable "Private Hilfe pro Kopf“}

Die Daten aus Punkt 1 werden durch die Anzahl Einwohner der Entwicklungs- und Transitionsländer geteilt.

3. Der Entwicklungstand eines bestimmten Landes -> Unabhängige Variable „Entwicklungsstand"

Zur Operationalisierung des Konzeptes verwenden wir den Synthetischen Indikator (zusammengesetzt aus Lebenserwartung, Bildung und Kaufkraft pro Einwohner) der UNO über den Stand der „menschlichen Entwicklung" der entsprechenden Länder von 0 (sehr tiefe menschliche Entwicklung) bis 1 (sehr hohe menschliche Entwicklung) aus dem Jahr $2000^{25}$.

4. Die humanitäre Lage eines Landes $\rightarrow$ zusammengesetzt aus den unabhängigen Variablen "Kriege", "Naturkatastrophen" und „Unterernährung".

4.1 Operationalisierung „Kriege“

Zur Messung der Schwere eines Konfliktes verwenden wir die Daten über Kriegsopferzahlen der Jahre 1997 bis 2001 des schwedischen Konfliktforschungsinstitutes SIPRI. Da die Daten teilweise unvollständig und ungenau sind, konstruieren wir einen Indikator für die Schwere eines bewaffneten Konfliktes von 0 (kein Konflikt) bis 4 (sehr schwerer bewaffneter Konflikt).

\subsection{Operationalisierung „Naturkatastrophen“}

Zur Messung der Schwere von Naturkatastrophen verwenden wir die Daten über Opferzahlen von Naturkatastrophen des Rückversicherungskonzerns SwissRe der Jahre 1998 bis $2001^{26}$. Die Opferzahlen der Jahre 1998, 1999 erhielten nur das halbe Gewicht, da wir annehmen, dass ein Teil des Wiederaufbaus bereits vor dem Jahr 2001 geleistet wurde. Auch die Katastrophen der zweiten Jahreshälfte 2001 erhielten nur das halbe Gewicht, da wir annehmen, dass der Wiederaufbau (und damit der Geldfluss) erst mit einer gewissen Verzögerung beginnt.

\subsection{Operationalisierung „Unterernährung“}

Wir verwenden die von der Welternährungsorganisation FAO erhobenen Daten über die Zahl der unterernährten Menschen aus der Messperiode 1999/200127.

5. Die Höhe der Hilfe, die ein Land aus dem Ausland erhält $\rightarrow$ Unabhängige Variable "Modeland"

Zur Operationalisierung verwenden wir die von der OECD erhobenen Zahlen aus dem Jahr 2001 über die gesamten Hilfeleistungen, welche ein entsprechendes Entwicklungs- oder Transitionsland aus dem Ausland erhält28.

24 DEZA, Aide de la Suisse aux pays en développement et aux pays en transition - statistiques 2001, DEZA, Bern, 2003.

25 PNUD, Rapport mondial sur le développement humain 2002, Editions De Boeck Université, Brüssel, 2002.

26 Schweizerische Rückversicherungsgesellschaft (Hg.), sigma Natur- und Man made-Katastrophen, Jahre 1998-2001, Zürich.

27 FAO, The State of Food Insecurity in the World 2003, Rom, 2003.

28 OECD, Statistical Annex of the 2003 Development Cooperation Report, Paris, Januar 2004. 
6. Aus Entwicklungs- und Transitionsländern in die Schweiz eingewanderte Personen $\rightarrow$ Unabhängige Variable „eingewanderte Wohnbevölkerung"

Zur Operationalisierung verwenden wir die vom Bundesamt für Statistik anlässlich der Volkszählung 2000 erhobenen Angaben über die Herkunft der Schweizer Wohnbevölkerung²9.

7. Staatliche Hilfeleistungen in Entwicklungs- und Transitionsländer $\rightarrow$ Unabhängige Variable "öffentliche Hilfe"

Zur Operationalisierung verwenden wir die von der DEZA erhobenen gesamten aus dem öffentlichen Sektor der Schweiz stammenden bilateralen Finanzströme aus dem Jahr 2001 (zusammengesetzt aus öffentlicher Entwicklungshilfe und öffentlicher Hilfe) ${ }^{30}$.

\section{B) Regressionen ${ }^{31}$}

1. Entwicklungsstand eines Landes

Abhängige Variable „Private Hilfe pro Kopf"

Unabhängige Variable "Entwicklungsstand“

$R=0.094 r^{2}=0.009$ Keine statistische Signifikanz

2. Soziale Notlagen und humanitäre Aspekte

Abhängige Variable: „Private Hilfe“

Unabhängige Variablen: „Kriege“ "Unterernährung“

$R=0.488 \quad r^{2}=0.238 \quad$ Statistische Signifikanz auf der $1 \%$-Ebene

„Kriege": $\quad$ Beta $=0.249 \quad$ Statistische Signifikanz auf der $1 \%$-Ebene

"Unterernährung": Beta $=0.365 \quad$ Statistische Signifikanz auf der $1 \%$-Ebene

3. Die "Modeländer“ der internationalen Hilfe

Abhängige Variable: „Private Hilfe“

Unabhängige Variable: „Modeland“

$R=0.512 \quad r^{2}=0.263 \quad$ Statistische Signifikanz auf der $1 \%$-Ebene

\section{Eingewanderte Wohnbevölkerung aus Entwicklungsländern}

Abhängige Variable: „Private Hilfe“

Unabhängige Variable: „Ausländische Wohnbevölkerung“

$R=0.526 \quad r^{2}=0.277 \quad$ Statistische Signifikanz auf der $1 \%$-Ebene

5. Beziehung Hilfswerke - Bund (DEZA)

Abhängige Variable: „Private Hilfe“

Unabhängige Variable: „Öffentliche Hilfe“

$R=0.711 \quad r^{2}=0.506 \quad$ Statistische Signifikanz auf der $1 \%$-Ebene

\section{Synthese}

Abhängige Variable: „Private Hilfe“

Unabhängige Variablen: „Öffentliche Hilfe“, „eingewanderte Wohnbevölkerung“, „Unterernährung”

$\mathrm{R}=0.768 \quad \mathrm{r}^{2}=0.590 \quad$ Statistische Signifikanz auf der $1 \%$-Ebene

"Öffentliche Hilfe" Beta $=0.475 \quad$ Statistische Signifikanz auf der $1 \%$-Ebene

"eingew. Wohnbev." Beta $=0.277 \quad$ Statistische Signifikanz auf der $1 \%$-Ebene

„Unterernährung" Beta $=0.252 \quad$ Statistische Signifikanz auf der $1 \%$-Ebene

$31 \quad R=$ Korrelationskoeffizient, $r^{2}=$ lineares Bestimmtheitsmass, Beta = standardisierter Regressionskoeffizient (zeigt die Bedeutung der entsprechenden unabhängigen Variable auf das Gesamtmodell an). 


\section{C) Tabellen}

Die Länder in Fettdruck befinden sich ebenfalls unter den 20 Ländern, welche die höchsten privaten Hilfeleistungen erhalten (Tabelle 1 "absolute Beiträge" für Tabellen 4, 5, 6 und Tabelle 2 „Pro-Kopf-Beiträge" für Tabelle 3).

Tabelle 3: „Entwicklungsstand“, die 20 am wenigsten entwickelten Länder (Human Development Index, 2000)

\begin{tabular}{llll}
\hline 1. Sierra Leone & 0.275 & 11. Zentralafrik. Rep. & 0.375 \\
\hline 2. Niger & 0.277 & 12. Mali & 0.386 \\
\hline 3. Afghanistan* & 0.300 & 13. Liberia* & 0.400 \\
\hline 4. Burundi & 0.313 & 14. Malawi & 0.400 \\
\hline 5. Mosambik & 0.322 & 15. Angola & 0.403 \\
\hline 6. Burkina Faso & 0.325 & 16. Ruanda & 0.403 \\
\hline 7. Äthiopien & 0.327 & 17. Gambia & 0.405 \\
\hline 8. Guinea-Bissau & 0.349 & 18. Guinea & 0.414 \\
\hline 9. Somalia* & 0.350 & 19. Benin & 0.420 \\
\hline 10. Tschad & 0.365 & 20. Eritrea & 0.421 \\
\hline
\end{tabular}

* eigene Schätzung.

Tabelle 4: „Öffentliche Hilfe“, die 20 Länder, welche am meisten öffentliche Entwicklungshilfe erhalten (in Tausend Franken, 2001)

\begin{tabular}{|c|c|c|c|c|}
\hline & Jugoslawien & 57741 & 11. Bolivien & 17979 \\
\hline 2. & Mosambik & 39393 & 12. Russland & 15112 \\
\hline 3. & Indien & 37399 & 13. Peru & 15095 \\
\hline 4. & Tansania & 28725 & 14. Pakistan & 15046 \\
\hline 5. & Burkina Faso & 21687 & 15. Südafrika & 13552 \\
\hline 6. & Bosnien-Herzegowina & 21078 & 16. Afghanistan & 12771 \\
\hline 7. & Nepal & 20912 & 17. Niger & 12378 \\
\hline 8. & Bangladesch & 20790 & 18. Tschad & 12027 \\
\hline 9. & Bulgarien & 18869 & 19. Tadschikistan & 11765 \\
\hline & Vietnam & 18262 & 20. Ecuador & 11688 \\
\hline
\end{tabular}

Tabelle 5: „Eingewanderte Wohnbevölkerung“, die 20 wichtigsten Herkunftsländer von Migranten (in Anzahl Personen, 2000)

\begin{tabular}{|c|c|c|c|}
\hline 1. Jugoslawien & 213524 & 11. Thailand & 5722 \\
\hline 2. Türkei & 83312 & 12. Marokko & 5680 \\
\hline 3. Mazedonien & 55714 & 13. Philippinen & 4986 \\
\hline Bosnien-Herzegowina & 47286 & 14. Vietnam & 4791 \\
\hline 5. Kroatien & 43084 & 15. Somalia & 4764 \\
\hline 6. Sri Lanka & 32708 & 16. Polen & 4689 \\
\hline 7. Brasilien & 8611 & 17. Irak & 4567 \\
\hline 8. Russland & 6812 & 18. Dominikanische Rep. & 4486 \\
\hline 9. China & 6315 & 19. Tunesien & 4418 \\
\hline Indien & 6142 & 20. Kongo, Rep Dem. & 4168 \\
\hline
\end{tabular}


Tabelle 6: „Unterernährung“, die 20 von Unterernährung am meisten betroffenen Länder (in Millionen unterernährter Menschen, 1999-2001)

\begin{tabular}{|c|c|c|c|}
\hline 1. Indien & 213.7 & 11. Vietnam & 15.1 \\
\hline 2. China & 135.3 & 12. Indonesien & 12.6 \\
\hline 3. Bangladesch & 44.1 & 13. Thailand & 11.9 \\
\hline 4. Kongo, Rep Dem. & 38.3 & 14. Kenia & 11.5 \\
\hline 5. Pakistan & 26.8 & 15. Mosambik & 9.7 \\
\hline 6. Äthiopien & 26.4 & 16. Nigeria & 9.1 \\
\hline 7. Philippinen & 16.8 & 17. Sudan & 7.7 \\
\hline 8. Brasilien & 15.6 & 18. Korea, Dem Rep & 7.5 \\
\hline 9. Afghanistan & 15.3 & 19. Angola & 6.4 \\
\hline 10. Tansania & 15.2 & 20. Usbekistan & 6.4 \\
\hline
\end{tabular}

